\title{
COLLIDER PERFORMANCE WITH IDEAL COLLISIONS
}

M. Wonth

BNL -51909

DE85 017011

\section{June 1, 1985}

\author{
DISCLAIMER
}

This report was prepared as an account of work sponsored by an ageticy of the United States Government. Neither the United States Government nor any ageacy thereof, nor any of their employees, rakes any warranty, express or implied, or asumes any legel liability or responsibility for the accuracy, completeness, or usefulness of any information, apparatus, product, or process disclosed, or represents that its use woukd not infringe privately owned rights. Reference herein to any specific commercial product, process, or service by trade name, trademark, manufacturer, or otherwis: does not neceasarily constitute or imply its endorsement, recommendation, or favoring by the United States Government or any avency thereof. The views and opinions of authors exprewed berein do not wecessarily state or rellect those of the United States Government or any agency thereof.

\section{ALTERMATING GRADIENT SYMCHROTROM DEPARTMENT}

\section{BROOKHAVEN NATIONAL LABORATORY UPTON, LONG ISLAND, NEW YORK 11973}




\title{
Collider Perfornance with Ideal Collieione
}

\author{
M. Month \\ June 1, 1985
}

\section{Sumery}

Performance is estianted for head-on bunch collisions. The luminosity is written as a distribution in time and length along the collision path, using a Gaussian distribution in all space dimensions. Computetions are ande for the Tevatron. Sources of luminosity reduction are listed in the case of non-ideal conditions.

\section{Luminosity}

The luminosity, L, is a messure of the event rate for a given perticle interaction cross section. If $\dot{n}$ is the number of events per. unit time for a given type of collision, described by the cross section $\sigma$, then we have

$$
\dot{\mathrm{n}}=\sigma \mathrm{L} \text {. }
$$

The luminosity is a relativistic invariant, and can be expressed by the over lap integral,

$$
L=c\left(\left|\vec{B}_{1}-\vec{B}_{2}\right|^{2}-\left|\vec{B}_{1} \times \vec{B}_{2}\right|^{2}\right)^{\frac{1}{2}} \int \rho(1) \rho(2) d \nabla,
$$

where $\rho(1)$ and $\rho(2)$ are volume particle densities, $\vec{B}_{1}$ and $\vec{B}_{2}$ are particle velocities in units of the velocity of light, $c$, and $d v$ is the space differentiel,

$$
d v=d x d y d z .
$$




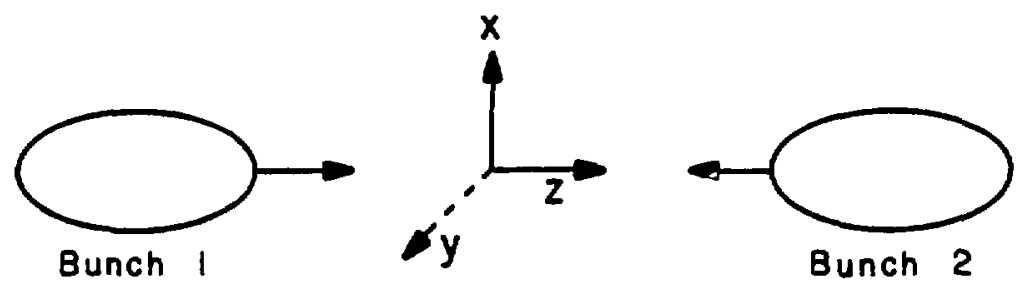

Figure 1: Head-on Collision Geometry.

For a Gaussian beam in all three space dimensions and in a coordinate system with the bunches moving in the a-direction, the densities can be written,

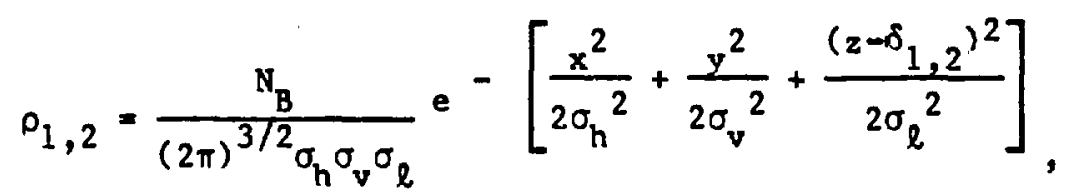

where $N_{B}$ is the number of particles in each bunch (taken for simplicity to be the same), $\sigma_{h}, \sigma_{v}, \sigma_{\ell}$ are horizontal, vertical and longitudinal rms bunch sizes at the crossing point, and $\delta_{1,2}$ are beam offsets for bunches 1 and 2 respective$1 y$.

To specify $\delta_{1,2}$, let bunch 1 be centered at the "collision point" at $t=0$, and let burich 2 be delayed by the $t$ ime $t_{0}$. Then,

$$
\delta_{1}=c t \quad, \quad \delta_{2}=-c\left(t-t_{0}\right)
$$

Substitute $\rho_{1,2}$ and $\delta_{1,2}$ into the general expression for the luminosity to obtain the instantaneous luminosity, $L(t)$. Then, integrate over time as the bunches pass through each other to find the luminosity per bunch collision, $L_{B}$ $\left(c m^{-2}\right)$ : 


$$
I_{B}=\int_{-\infty}^{\infty} L(t) d t .
$$

Problem: Assuming that the $\sigma^{\prime}$ s are independent of $z$, show that $L_{B}$ is independent of the offset, $c_{0}{ }^{*}$

Problem: Show, in the round beam case, $\sigma_{h}=\sigma_{v}=\sigma$, that $\mathrm{L}_{B}$ is independent of $\sigma_{\ell}$ and is given by,

$$
L_{B}=\frac{N_{B}^{2}}{4 \pi r^{2}}
$$

Define the transverse emittance in each dimension as the phase space area containing $95 \%$ of the particles in the beam. Denote this emittance by $\varepsilon$, which can be written in terms of the normalized emittance $\mathbf{E}$, by

$$
\varepsilon=E / B Y
$$

For a Gaussian beam, $\sigma^{2}$ at the crossing point can be expressed in terms of $E$ by

$$
\sigma^{2}=\frac{E B \star}{6 \pi Y}
$$

where $B *$ is the $B$ value at the crossing point and the velocity, $B$, has been taken to be 1 .

Proof: In terms of the normalized coordinates, $\eta=x / \sqrt{B \star}, \dot{n}=\sqrt{B^{\star}} x^{\prime}$, a Gaussian phase space density is given by

$$
\rho(\text { phase space })=\frac{B \star}{2 \pi \sigma^{2}} \mathrm{e}^{-\left(\eta^{2}+\eta^{2}\right) /\left(2 \sigma^{2} / B \star\right)},
$$

where, 


$$
\text { Sodnd } \dot{n}=1
$$

Transforming to Polar coordinates: $r$, $\phi$, we have

$$
\rho\left(r^{2}\right)=\frac{B *}{2 \pi \sigma^{2}} e^{-r^{2} /\left(2 \sigma^{2} / B *\right)} .
$$

Let $R$ be the "95\% radius," then

$$
\int_{0}^{2 \pi} \int_{0}^{R} r d r d \phi \rho\left(r^{2}\right)=0.95
$$

Integrating leads to the equation

$$
\left[1-\mathrm{e}^{-\mathrm{R}^{2} /\left(2 \sigma^{2} / R *\right)}\right]=0.95 \text {. }
$$

Thus, the area,

$$
\pi R^{2}=\frac{E}{\gamma}=\frac{2 \pi \sigma^{2}}{B^{*}} \ln (20)=\frac{6 \pi \sigma^{2}}{B^{*}}
$$

Using the expression for $\sigma^{2}$, we have the luminosity per bunch collision,

$$
L_{B}=\frac{3}{2} \frac{\mathrm{N}_{B}^{2} \gamma}{\mathrm{E}^{*}} \cdot\left(\text { units } \mathrm{cm}^{-2}\right) \text {. }
$$

The average luminosity is $L_{B}$ times the bunch collision frequency, $f_{B}$ :

$$
L_{A V}=L_{B} f_{B} \text {, }
$$

or, 


$$
L_{A V}=\frac{3}{2} \frac{N_{B}{ }^{2} E_{B} \gamma}{E B *} \cdot\left(\text { units } c^{-2} \sec ^{-1}\right)
$$

Time and Space Distribution of Luminosity

In the case where $\sigma_{h}$ and $\sigma_{v}$ are independent of $z$ in the crossing region (i.e. $B_{h, v}^{*} \gg \sigma_{\ell}$ ), then the instantaneous luminosity can be expressed as anction of length along the bunch collision path, $z$, and time, $t$. Using the expreasions for $\delta_{1,2}$ in terms of $t$ and integrating over the transverse cross section (i.e. over $x$ and $y$ ), we can show that

$$
L(t, z)=\frac{c_{B}^{2}}{(2 \pi)^{2} \sigma_{\ell}{ }^{2} \sigma_{h} \sigma_{v}} e^{-\left(z-d_{2} c t_{0}\right) 2 / \sigma_{l}{ }^{2}} e^{-c^{2}\left(t-d_{z} t_{0}\right) 2 / \sigma_{l}{ }^{2}} .
$$

Introducing the dimensionless variables

$$
s=2 / \sigma_{\ell} \text { and } \tau=c t / \sigma_{\ell} \text { and }
$$

writing the dimensionless time offset, $\tau_{0}=c t_{0} / \sigma_{\ell}$, we have for the specific instantaneous luminosity

$$
\frac{d Q(\tau, s)}{d \tau d s}=\frac{N_{B}^{2}}{4 \pi^{2} \sigma_{h} \sigma_{v}} e^{-\left(s-\frac{1}{2} \tau_{0}\right)^{2}} e^{-\left(\tau-\frac{d_{2}}{2} \tau_{0}\right)^{2}}
$$

where $\mathscr{L}(\tau, s)$ is a measure of the luminosity per bunch collision. For ideal collisions, that is, with zero offset,

$$
\frac{d G(\tau, s)}{d \tau d s}=\frac{N_{B}^{2}}{4 \pi^{2} \sigma_{h} \sigma_{v}} e^{-s^{2}} e^{-\tau^{2}}
$$


Integracing over space and time gives the luminosity per bunch collision,

$$
L_{B}=\frac{N_{B}^{2}}{4 \pi \sigma_{h} \sigma_{v}} .
$$

Thus,

$$
\frac{\mathrm{d} \mathscr{L}}{\mathrm{d \tau d} a}=\frac{\mathrm{L}_{\mathrm{B}}}{\pi} \mathrm{e}^{-\mathrm{s}^{2}} \mathrm{e}^{-\tau^{2}}
$$

Luminosity Definitions

$$
\begin{aligned}
& \frac{\mathrm{d} \mathscr{G} \tau, s)}{\mathrm{d} \tau \mathrm{ds}} \quad \text { Spacific instantaneous luminosity } \\
& \frac{\mathrm{d} \mathscr{P}}{\mathrm{dt}} \quad \text { Instantaneous luminosity } \\
& \frac{d \mathscr{Q}}{\mathrm{ds}} \quad \text { Specific luminosity } \\
& \mathrm{L}_{B} \equiv \mathscr{Q} \quad \text { Luninosity per bunch collision } \\
& \mathrm{L}_{\mathrm{AV}}=\mathscr{L}_{\mathrm{B}} \quad \text { Average luminosity } \\
& \text { ( } f_{B} \text { is the frequency of bunch collisions). }
\end{aligned}
$$

Idea 1 Tevatron Luminosity

To compute the Tevatron performance with ideal collisions, we need the characteristics of the colliding bunckes. Ideal collisions are characterized by having the proton and antiproton bunches identical. However, it is quite possible that as the Tevatron collider gets started, the antiproton bunch intensity will be significantly lower than the proton bunch intensity. To modify our expressions to take account of this difference, we simply take



The Tevatron parameters for the assumed identical bunches are as follows: 


\section{Longitudinal}

rms bunch length: $\sigma_{l}=40 \mathrm{~cm}$

957 bunch length: $\quad \ell_{B} \doteq 2 \sqrt{6} \sigma_{\ell}$

rms momentum speed: $\sigma_{p} / p=1.2 \times 10^{-4}$

\section{Transverse}

rns width at crossing point:

$$
\sigma^{*}=\left[\frac{\beta * \mathrm{E}}{6 \pi \gamma}\right]^{\frac{1}{2}}=6.1 \times 10^{-5} \mathrm{~m} .
$$

Taking, $\beta$ at the crossing point: $\beta *=1 \mathrm{~m}$

Energy in mass units: $\quad Y=1066.1$ ( $1 \mathrm{TeV}$ )

and the normalized 957 emittance: $\quad E=24 \pi \times 10^{-6} \mathrm{rad}-\mathrm{mL}$

rms horizontal and vertical widths: $\sigma_{v}=\sigma_{h}=\sigma *=6.1 \times 10^{-5} \mathrm{~m}$.

Thus, assuming the number of particles per bunch is the same for protons and antiprotons,

$$
N_{p}=N_{p}=6 \times 10^{10}
$$

we obtain for the luminosity per bunch collision,

$$
L_{B}=\frac{N_{p} N_{p}}{4 \pi \sigma_{h} \sigma_{v}}=0.76 \times 10^{25} \mathrm{~cm}^{-2} .
$$

Since the revolution frequency at relativistic velocities is $\mathrm{E}_{0}=4.77 \times 10^{4} \mathrm{Ez}$, we have, taking $n_{B}=3$ bunches in each beam, for the frequency of bunch collisions,

$$
f_{B}=n_{B} f_{0}=143.1 \mathrm{kHz}
$$

Thus the average luminosity,

$$
L_{A V}=f_{B} L_{B}=1.1 \times 10^{30} \mathrm{~cm}^{-2} \mathrm{sec}^{-1}
$$


Also, it should be noted that with a total interaction cross section of $\sigma_{\text {tot }}$

- $100 \mathrm{mb}$, the mean number of everts per bunch collision is

$$
n_{\text {mean }}=\mathrm{L}_{B} \sigma_{\text {tot }}=0.76 \text {. }
$$

The specific instantaneous luminosity is given by

$$
\frac{d \mathscr{C}}{d \tau d s}=\frac{L_{B}}{\pi} e^{-\tau^{2}} e^{-s^{2}}
$$

where we have that

$s$ is a normalized length: $s=2 / \sigma_{\ell}$

$\tau$ is a nomalized time: $\tau=c t / \sigma_{\ell}$.

Thus, the luminosity distribution is characterized by:

rms length of luminosity along collision path

$$
=\frac{1}{\sqrt{2}} \sigma_{\ell}=28.3 \mathrm{~cm}
$$

rms time distribution of luminosity within a bunch collision

$$
=\frac{1}{\sqrt{2}} \frac{\sigma_{\ell}}{c}=0.94 \mathrm{nsec}
$$

(note that the period of bunch collisions

$$
\left.=\frac{1}{f_{B}}=7.0 \text { usec }\right) \text {. }
$$

\section{Sources of Luminosity Reduction}

The luminosity calculated with an ideal collision configuration will in practice be reduced because actual conditions may nut conform to the ideal. A list of some of the sources of luminosity reduction is as follows: 
1. If $\sigma_{\ell} \geqslant 8 *$, we must mitiply the overlap density by the factor

$$
\left[\left(1+\frac{s^{2} \sigma_{\ell}^{2}}{\beta_{h}^{* 2}}\right)\left(1+\frac{s^{2} \sigma_{\ell}^{2}}{\beta_{v}^{2}}\right)\right]^{\alpha_{2}}
$$

This is related to the fact that even when the bunch centers weet at the collision point, if the bunches are "long," then the ends of the bunch are simultaneously at points of higher beta and therefore points of lower overlap density .

2. If there is a delay of bunch 2 with respect to bunch 1 , it will, if uncorrected, result in a luminosity reduction, again because of an effective reduction in overlap density.

3. A redustion of luminosity will result if there is an offset of the minimum of $\beta_{h}^{*}$ with respect to $B_{v}^{*}$, even if they are equal in magnitude.

4. An increase of emittance of beam 1 with respect to beam 2 will result in a luminosity reduction and should be considered. In the SPS, it appears that the low intensity beam (the weak beam) cannot sustain an emittance larger than the strong beam due to the electromagnetic beam-beam effect. Thus in the weakstrong case, the emittances tend to equalize if the weak beam emittance is initially greater than the strong beam emittance. of course the number of particles in the weak beam is reduced.

5. If the dispersion function is not zero in the collision region, the overlap density is reduced.

6. There are phenomena which decrease the luminosity lifetime by either increasing beam bunch sizes $\left(\sigma_{h}, \sigma_{v}\right.$, or $\left.\sigma_{l}\right)$ or by decreasing the number of particles in the bunches. Size incresse can be caused by scattering off the residual gas in the vacuum chamber; intrabeam scattering; blowup due to the bean- 
beam interaction; and blow p due to the interaction of the environment with intense bunches. The wont important of these are probably intrabenm scattering in the intense proton bunches and beam-beam blowup of the "weak" antiproton bunches. Direct particle loss will occur mainly from events arising from the total nuclear $\mathrm{p} \overline{\mathrm{p}}$ cross section. As was seen earlier, using a total cross secion of $100 \mathrm{mb}$, the mean evert rate is 0.76 events per bunch collision. In the case of high luminosity together with low bunch current, this could become a limiting factor. In the Tevatron, however, this should not be a problem.

10 